\title{
Cirugía pancreatobiliar laparoscópica: experiencia inicial en el hospital regional de alta especialidad
}

\section{Laparoscopic pancreatobiliar surgery: Initial experience in high speciality regional hospital}

\author{
Gustavo A. Guerrero-Martínez *, Armando Basilio-Roque³, Roberto Estrada-Gómez²,
} Jorge A. Roldán-García ${ }^{1}$, Rafael Viveros-Luna ${ }^{3}$ y Pablo A. Dávila-Esparza ${ }^{1}$

'Servicio de Cirugía General y Laparoscopía, Hospital de Alta Especialidad, Instituto de Seguridad y Servicios Sociales de los Trabajadores del Estado (ISSSTE) Puebla; '2Servicio de Cirugía General, Hospital Ángeles Puebla; ${ }^{3}$ Servicio de Oncología Quirúrgica, Hospital de Alta Especialidad, Instituto de Seguridad y Servicios Sociales de los Trabajadores del Estado (ISSSTE) Puebla. Puebla, Puebla, México

\section{Resumen}

Antecedentes: En la actualidad las enfermedades benignas y malignas de las vías biliares y del páncreas requieren un manejo multidisciplinario, y las técnicas para resolver este tipo de padecimientos han avanzado gracias al mejoramiento en la tecnología y la capacidad del personal humano. Objetivo: El propósito de este trabajo es presentar varios casos y demostrar que la cirugía de mínima invasión en patología benigna y maligna pancreatobiliar es viable y se pueden obtener adecuados resultados. Método: De julio de 2013 a julio de 2018, 30 pacientes consecutivos han sido intervenidos quirúrgicamente por vía laparoscópica por diversas enfermedades que involucran páncreas y vías biliares, por el mismo cirujano y equipo quirúrgico, con un seguimiento de hasta 4 años. Resultados: Ninguno de los pacientes intervenidos presentó complicaciones graves en el transoperatorio ni en el posquirúrgico inmediato, ninguno fue sometido a una segunda cirugía y los tiempos quirúrgicos y el sangrado fueron aceptables. Conclusión: Los resultados son positivos y muy alentadores, ya que la morbilidad fue muy similar a la de las series que se revisaron; sin embargo, este estudio es nuestra experiencia inicial y requerimos aumentar el número de casos para obtener una muestra significativa.

Palabras clave: Cirugía pancreatobiliar laparoscópica. Cirugía de mínima invasión. Tumores benignos y malignos de páncreas y vías biliares. Coledocolitiasis. Enfermedades pancreatobiliares. Derivación biliodigestiva.

\begin{abstract}
Background: At present benign and malignant pancreatobiliar diseases, required a multidisciplinary management, and the different techniques to solve this pathology has been improve with new technology and surgeons training. Objective: The purpose of this article is to describe that de minimally invasive surgery on benign and malignant pancreatobiliar diseases is feasible and offer good results. Method: In Hospital regional ISSSTE Puebla from July 2013 to July 2018, 30 consecutive patients with different pancreas and biliary pathologies have been operated by totally laparoscopic, by the same surgeon and surgical team, and following for up to 4 years. Results: None all the patients presented sever complications during surgery or after immediate surgery and no reoperations. Surgical time and blood loss was acceptable.
\end{abstract}

\section{Correspondencia:}

*Gustavo A. Guerrero-Martínez

Privada de las Ramblas, 4

Col. Desarrollo Atlixcáyotil

Fecha de recepción: 18-01-2018

Cir Cir. 2020;88(6):679-683 
Conclusion: The results we obtained are positive and encouraging because the morbidity and mortality are similar to the different sources we reviewed, nevertheless, this study is our initial experience and we required more cases to obtain a significant sample.

Key words: Malignant and benignant pancreatobiliar diseases. Advanced laparoscopy. Laparoscopic pancreatobiliar surgery. Choledocholitiasis. Biliodigestive bypass. Biliopancreatic tumors.

\section{Introducción}

La patología pancreatobiliar tiene un espectro muy amplio de enfermedades, tanto benignas como malignas, que requieren un manejo altamente especializado y multidisciplinario ${ }^{1,2}$. Desde los años 1990, cuando inició la cirugía laparoscópica en México, se han realizado diversos procedimientos quirúrgicos laparoscópicos con buenos resultados. Las resecciones pancreáticas iniciaron en esa misma década principalmente por patología benigna $a^{3,4}$ y más tarde se presentaron los primeros reportes de cirugía pancreática por enfermedad maligna, que al principio se limitaba a la estadificación o toma de biopsias y más tarde llegó a resecciones mayores. La cirugía de vías biliares por enfermedad obstructiva benigna o maligna ha tomado fuerza y existen diversos procedimientos derivativos que se efectúan por cirugía de mínima invasión, con una disminución muy importante de la morbilidad comparada con la cirugía convencional ${ }^{5-7}$, desde el tratamiento puramente paliativo hasta el tratamiento resolutivo en el caso específico de la enfermedad benigna ${ }^{8,9}$. La cirugía pancreatobiliar laparoscópica ha tenido mayor aceptación conforme se han obtenido mejores resultados en tiempos quirúrgicos, morbilidad, mortalidad y menor trauma quirúrgico en comparación con la cirugía abierta, además de ofrecer las ventajas de la cirugía mínimamente invasiva ${ }^{10,11}$.

\section{Método}

Desde julio de 2013 hasta febrero de 2018, en el Hospital Regional de Alta Especialidad del Instituto de Seguridad y Servicios Sociales de los Trabajadores del Estado (ISSSTE) Puebla se han realizado diferentes procedimientos laparoscópicos que involucran páncreas y vías biliares por patología tanto benigna como maligna, con buenos resultados. Se presentan 30 casos de pacientes a quienes se les realizaron diversos procedimientos, dentro de los que destacan derivaciones biliodigestivas y resecciones pancreáticas por diversas causas.
Tabla 1. Tipo de patologías resueltas por laparoscopía en el Hospital Regional de Alta Especialidad del ISSSTE Puebla

\begin{tabular}{lc}
\hline Patología & Pacientes \\
\hline Coledocolitiasis & 11 \\
Quiste de colédoco & 2 \\
Síndrome de Mirizzi & 1 \\
Lesión de vía biliar & 3 \\
Ampuloma & 3 \\
Tumor pseudopapilar de cola y cabeza de páncreas & 2 \\
Adenocarcinoma de cabeza de páncreas & 7 \\
Síndrome de Lemmel & 1 \\
\hline
\end{tabular}

\section{Resultados}

De los 30 pacientes, 25 eran mujeres (82\%) y 5 hombres (18\%), con una edad entre 36 y 83 años (media: 65 años) (Tabla 1). A 12 pacientes (42\%) se les realizó coledocoduodenostomía (Fig. 1), 11 por coledocolitiasis primaria de grandes elementos no resuelta por esfinterotomía endoscócpica y uno por síndrome de Lemmel; a 10 pacientes (32\%) se les realizó hepatoyeyunostomía en $Y$ de Roux (Fig. 2), cuatro por adenocarcinoma de cabeza de páncreas con criterios de irresecabilidad y colocación de prótesis biliar endoscópica fallida, dos por quiste de colédoco tipo IVa y tipo I (Fig. 3), uno por síndrome de Mirizzi tipo IV y tres por lesión de vía biliar (Strasberg E2 y E3) (Figs. 4 y 5); siete pacientes (21\%) fueron sometidos a pancreatoduodenectomía por enfermedad maligna, tres por tumor maligno del ámpula de Vater (Fig. 6) y tres por cáncer de cabeza de páncreas, uno por tumor sólido pseudopapilar de cabeza de páncreas y una paciente a quien se le realizó pancreatectomía distal con preservación esplénica por tumor sólido pseudopapilar de cola de páncreas (Tabla 2).

De los pacientes con coledocoduodenoanastomosis, ninguno presentó complicaciones. De los pacientes con hepatoyeyunostomía en $Y$ de Roux, uno (adenocarcinoma de cabeza de páncreas) cursó con fístula biliar que requirió tratamiento médico y aporte nutricional para la resolución de la fístula. De los pacientes con procedimiento de Whipple, uno tuvo 


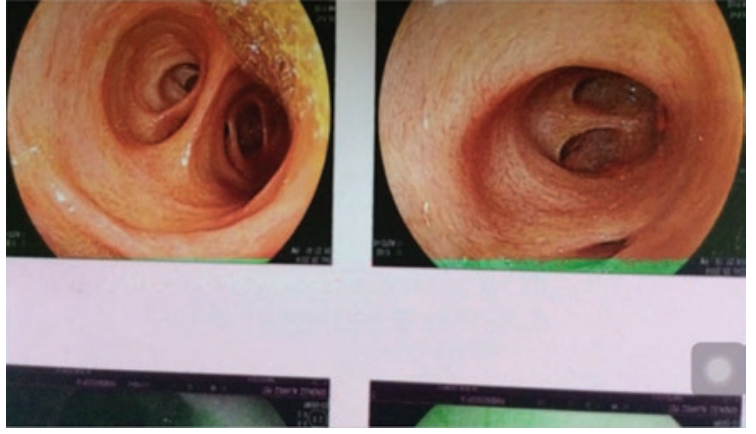

Figura 1. Control endoscópico posterior a coledocoduodenostomía.

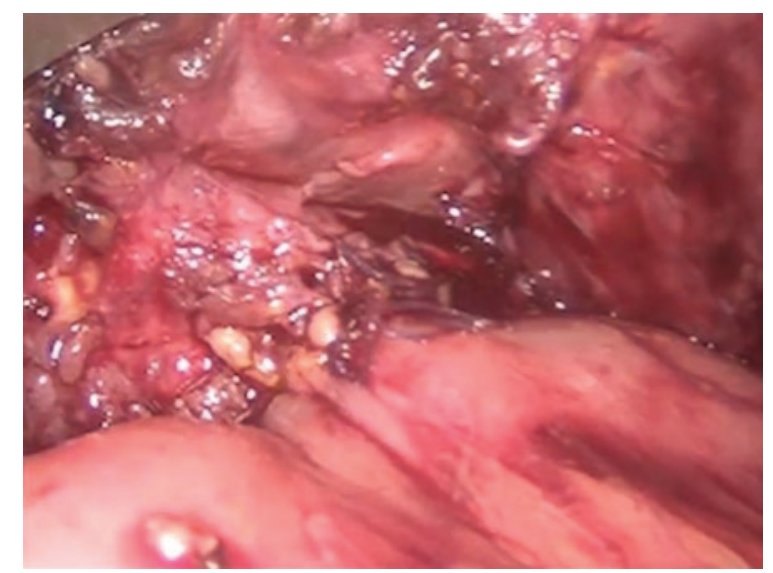

Figura 2. Hepatoyeyunostomía terminada posterior a lesión de vía biliar Strasberg E2.

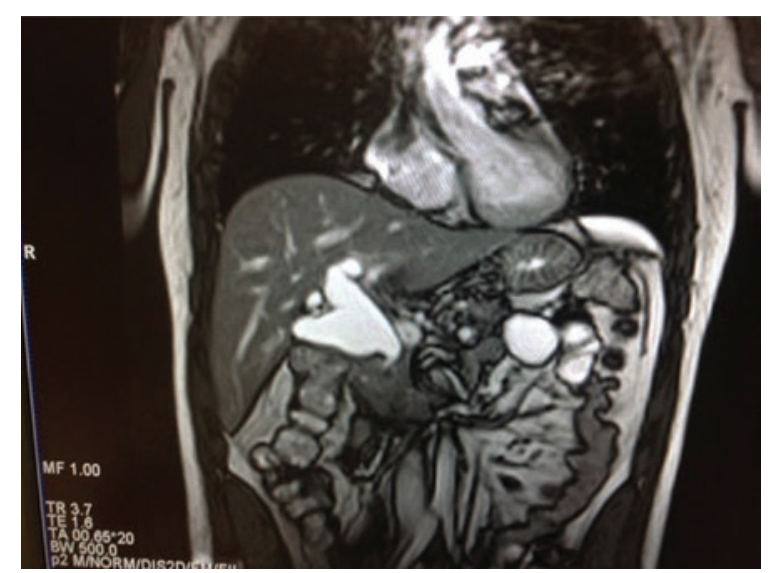

Figura 3. Resonancia magnética de un paciente con quiste de colédoco tipo IVa.

neumonía que requirió ingreso en la unidad de cuidados intensivos por 13 días en el posoperatorio, resolviéndose con tratamiento médico de manera exitosa, y otro más presentó sangrado transquirúrgico

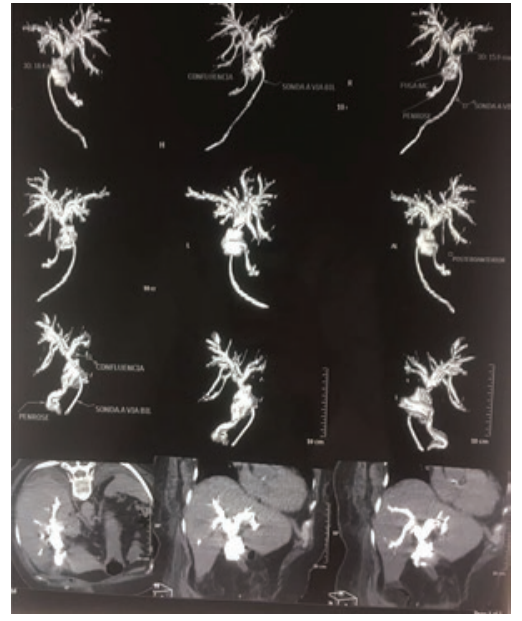

Figura 4. Colangiotomografía en la que se observa pérdida de continuidad de la vía biliar distal $2 \mathrm{~cm}$ por debajo de la confluencia de los hepáticos derecho e izquierdo (lesión de vía biliar Strasberg E2).

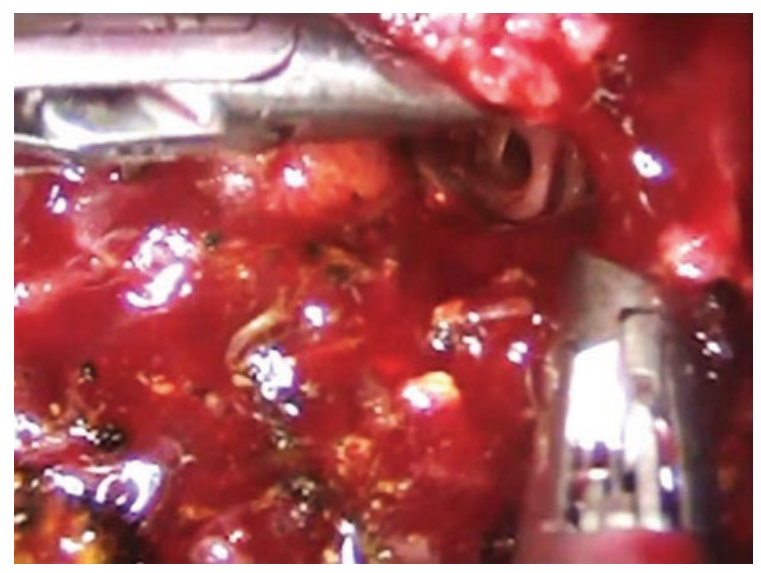

Figura 5. Vía biliar proximal disecada posterior a lesión de vía biliar Strasberg E2.

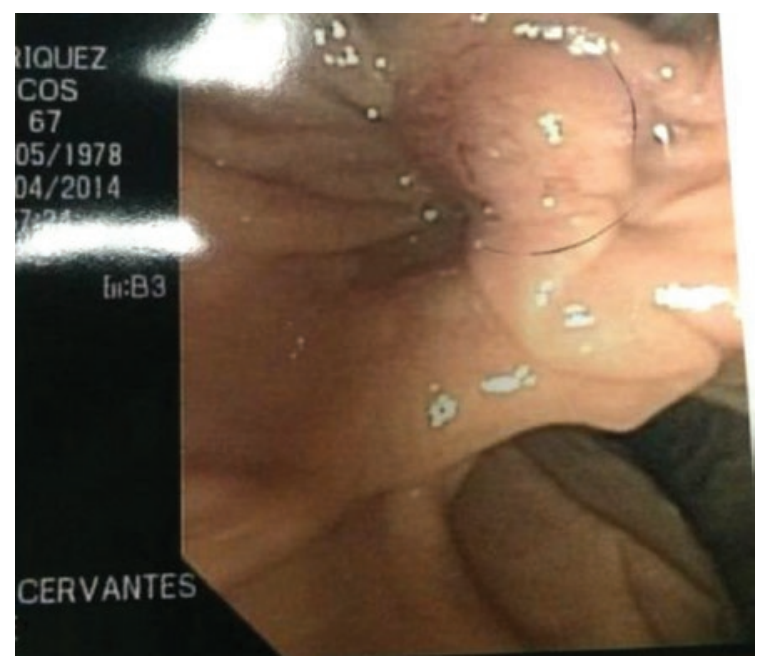

Figura 6. Duodenoscopía con hallazgo de ampuloma con toma de biopsia. 
Tabla 2. Procedimientos quirúrgicos realizados por laparoscopía

\begin{tabular}{lc}
\hline Cirugías & $\mathbf{n}$ \\
\hline Coledocoduodenostomía & 12 \\
Hepatoyeyunostomía en Y de Roux & 9 \\
Pancreatectomía distal con preservación esplénica & 1 \\
Pancreatoduodenectomía & 7 \\
\hline
\end{tabular}

moderado $(700 \mathrm{cc})$. Finalmente, un paciente con tumor de cola de páncreas a quien se realizó pancreatectomía distal con preservación esplénica no presentó complicaciones posquirúrgicas.

Ningún paciente presentó complicaciones graves en el transoperatorio ni en el posquirúrgico. Hubo conversión a cirugía tradicional en un paciente a quien se realizó pancreatoduodenectomía por sangrado transoperatorio que no se pudo controlar por mínima invasión, con buen posoperatorio. Ningún paciente fue sometido a reintervención. La estancia hospitalaria fue de 5 a 19 días, el sangrado transquirúrgico fue entre 100 y $1200 \mathrm{ml}$, y los tiempos quirúrgicos fueron de 3 a 9 horas.

\section{Discusión}

Desde los años 1990, cuando se realizaron la primera coledocoduodenostomía latero-lateral por patología benigna y posteriormente por enfermedad maligna, el bypass biliar (tanto colédoco-duodeno como hepático-yeyuno y anastomosis en $\mathrm{Y}$ de Roux) para este tipo de enfermedades ha tenido resultados muy favorables, por lo que se ha propuesto como manejo quirúrgico resolutivo de primera opción en lugar de la cirugía tradicional6,12.

El rol de la cirugía de mínima invasión en México inició con el tratamiento de enfermedades inflamatorias del páncreas, principalmente complicaciones de pancreatitis aguda como necrosectomías y cistogastroanastomosis ${ }^{3}$. Posteriormente, la estadificación y las resecciones parciales en enfermedades benignas y malignas se iniciaron con Bernheim, Cuschieri, Ishida y Meyer Burg, hasta que en 1994 se realizó la primera pancreatoduodenectomía laparoscópica en una mujer con pancreatitis crónica ${ }^{1,3}$. Desde entonces, la cirugía laparoscópica ha evolucionado de tal manera que prácticamente no existen contraindicaciones ni limitaciones en cuanto al tipo de patologías que se pueden resolver con esta técnica ${ }^{13,14}$. Se han reportado los resultados obtenidos en este tipo de procedimientos y la gran mayoría coinciden en que la técnica de mínima invasión en cirugía pancreatobiliar es altamente efectiva $a^{15,16}$, y principalmente para las resecciones pancreáticas distales con o sin preservación esplénica está tomando gran fuerza y en numerosos estudios es la primera opción para las enfermedades en esta región del páncreas ${ }^{17-21}$.

Si bien es cierto que la endoscopía intervencionista ha tenido excelentes resultados en el tratamiento de patología obstructiva, tanto benigna como maligna, en las enfermedades del páncreas y las vías biliares, la cirugía pancreatobiliar de mínima invasión ha demostrado ser muy efectiva en la resolución de los casos complejos no resueltos por endoscopía y en los pacientes con indicación quirúrgica, mostrando una morbimortalidad aceptable en comparación con la cirugía abierta y con resultados prometedores ${ }^{22}$. Tiene como limitante la complejidad técnica, que involucra resecciones de uno o más órganos, anastomosis del tracto digestivo y disecciones vasculares. Además, existen estudios que han hecho hincapié en que la adecuada selección del paciente es muy importante para obtener mejores resultados ${ }^{21,23}$.

En nuestro estudio se presentan 30 pacientes con diversas patologías que requirieron algún tipo de intervención quirúrgica, tanto endoscópica como por laparoscopía. La selección de los casos fue de acuerdo con el tipo de enfermedad, la edad, el estado general de los pacientes y el protocolo de estudio completo (imágenes, laboratorio, biopsias, etc.), ofreciendo en algunos casos la resolución del problema por vía endoscópica de acuerdo con las guías internacionales y, al no poder ser resueltas por este medio, se optó por la resolución endoscópica.

\section{Conclusión}

La cirugía pancreatobiliar de mínima invasión por enfermedad benigna o maligna es viable actualmente, y conforme existan más reportes y experiencia en todo el mundo irá ganando terreno y quizá en un futuro será la primera opción quirúrgica. Nuestro hospital es un centro de referencia que brinda atención a la población de todos los municipios del Estado de Puebla y de otros Estados de la república mexicana (Tlaxcala, Veracruz y Oaxaca), por lo que es necesario aumentar el tamaño de la muestra y continuar con el seguimiento a largo plazo de los pacientes.

Los resultados que obtuvimos son positivos y muy alentadores, ya que la morbilidad fue muy similar a la reportada en las series que se revisaron. Sin embargo, este estudio es nuestra experiencia inicial y 
requerimos continuar aumentando el número de casos para obtener una muestra significativa.

\section{Agradecimiento}

Al personal médico involucrado en el manejo integral de los pacientes.

\section{Conflicto de intereses}

Los autores declaran no tener conflicto de interés.

\section{Responsabilidades éticas}

Protección de personas y animales. Los autores declaran que para esta investigación no se han realizado experimentos en seres humanos ni en animales.

Confidencialidad de los datos. Los autores declaran que han seguido los protocolos de su centro de trabajo sobre la publicación de datos de pacientes.

Derecho a la privacidad y consentimiento informado. Los autores han obtenido el consentimiento informado de los pacientes y/o sujetos referidos en el artículo. Este documento obra en poder del autor de correspondencia.

\section{Bibliografía}

1. Alsfasser G, Hermeneit S. Minimally invasive surgery for pancreatic disease - current status. Dig Surg. 2016;33:276-83.

2. Fernández Cruz L, César-Borges G. Minimally invasive surgery of the pancreas in progress. Langenbecks Arch Surg. 2005;390:342-54.

3. Molina JF, Garnica G. Cirugía laparoscópica de patología pancreática. Cirujano General. 2010;32:25-6

4. Shimizu S, Tanaka M, Konomi H. Laparoscopic pancreatic surgery. Surg Endosc. 2004;18:402-6.
5. Sha B. Laparoscopic choledochoduodenostomy for retained bile duct stone. J Postgrade Med. 2005;51:156-7.

6. Olmedo I, Cuendis A. Coledocoduodenoanastomosis laparoscópica como opción terapéutica en coledocolitiasis compleja. Cir Cir. 2013;81:118-24.

7. Luu C, Lee B, Stabile BE. Choledochoduodenostomy as the biliary-enteric bypass of choice for bening and malignant distal common bile duct stricures. Am Surg. 2013;79:1054-7.

8. Chander J, Mangla V, Vindal A, Lal P, Ramteke VK. Laparoscopic choledochoduodenostomyy for biliary stone disease: a single-center 10 year experience. J Laparoendosc Adv Surg Tech A. 2012;22:81-4.

9. Khan AZ, Miles A. Initial experience with laparoscopic bypass for upper gastrointestinal malignancy: a new option for palliation of patients with advanced upper gastrointestinal tumors. J Laparoendosc Adv Surg Tech A. $2005 ; 15: 374-7$.

10. Brasesco O, Rossin D, Rosenthal J. Laparoscopic surgery of the liver and biliary tract. J Laparoendosc Adv Surg Tech A. 2002;12:91-100.

11. Giger U, Michel JM, Wiesli P. Laparoscopic surgery for benign lesions of the pancreas. J Laparoendosc Adv Surg Tech A. 2006;16:452-7.

12. Toumi Z, Aljarabah M, Ammori BJ. Role of the laparoscopic approach to biliary bypass for benign and malignant biliary diseases: a systematic review. Surg Endosc. 2011;25:2105-16.

13. Liao $\mathrm{CH}$, Yeh $\mathrm{CN}$, Wang SJ, Ouyang $\mathrm{CH}$. Effectiveness and feasibility of laparoscopic distal pancreatectomy on patients at high anesthetic risk. $J$ Laparoendosc Adv Surg Tech A. 2014;24:865-71.

14. Kang CM, Yang WY, Lee YH, Choi GH. A case of spleen-preserving laparoscopic distal pancreatectomy and concomitant cholecystectomy in male patient with solid pseudopapillary neoplasm of the pancreas and gallstone. J Laparoendosc Adv Surg Tech A. 2008;18:259-65.

15. Abu Hilal M, Hamdan M, Di Fabio F, Pearce NW, Johnson CD. Laparoscopic versus open distal pancreatectomy: a clinical and cost-effectiveness study. Surg Endosc. 2012;26:1670-4.

16. Corcione F, Pirozzi F, Cuccurullo D, Piccolboni D. Laparoscopic pancreatoduodenectomy: experience of 22 cases. Surg Endosc. 2013;27:2131-6.

17. Dulucq JL, Wintringer $P$, Stabilini $C$. Are major laparoscopic resections worthwhile? Surg Endosc. 2005;19:1028-34.

18. Sharad S, Doumane G, Mura T. Laparoscopic versus open distal pancreatectomy: a single institution case-control study. Surg Endosc. 2012; 26:402-7.

19. Schlöricke E, Nolde J, Hoffmann M, Roblick U, Bruch HP. Laparoscopic spleen-preserving distal pancreatectomy. Langenbecks Arch Surg. 2011;396:1119-23.

20. Kneuertz PJ, Patel SH, Chu CK, Fisher SB, Maithel SK, Sarmiento JM, et al. Laparoscopic distal pancreatectomy: trends and lessons learned through an 11-year experience. J Am Coll Surg. 2012;215:167-76.

21. Basuch D, Keck T. Laparoscopic pancreatic resections. Langenbecks Arch Surg. 2013;398:939-45.

22. Date RS, Siriwardena K. Current status of laparoscopic biliary bypass in the management of non-resectable peri-ampullary cancer. Pancreatology. 2005;5:325-9.

23. Wolfgang CL, Herman JM, Laheru DA, Klein AP, Erdek MA, Fishman EK, et al. Recent progress in pancreatic cancer. CA Cancer J Clin. 2013;63:318-48. 